con hojas ligeramente más anchas, (1)1,5-4(5) $\mathrm{mm}$, glabrescentes o esparcidamente pelosas al menos en una de sus caras y márgenes, y que está representada en la Península Ibérica fundamentalmente en el extremo oriental del Sistema Cantábrico y, más raramente, en los Pirineos orientales (Sierra del Cadí) [Esp.: B $\mathrm{Bu} \mathrm{Na}$ Vi]. Ambas variedades, además, difieren en la pilosidad del involucelo, ya que mientras que en la var. graminifolia los pelos del tubo a menudo alcanzan y solapan las fosetas, en la var. virescens no lo hacen.
AGRADECIMIENTOS. Nuestro sincero agradecimiento a los conservadores de los herbarios BC, GDA-GDAC, JAEN, MA, MGC, SEV y UNEX.

Aceptado para su publicación en octubre de 2003

Dirección de los autores. Unidad de Botánica, Departamento de Biología y Producción Vegetal, Facultad de Ciencias, Universidad de Extremadura. Avda. de Elvas s/n. 06071 Badajoz.

\title{
132. UNA NUEVA ASOCIACIÓN PREPIRENAICA DE ASPLENION PETRARCHAE BR.-BL. IN MEIER \& BR.-BL. 1934 NOM. MUT.
}

\author{
Manuel B. CRESPO, Ana JUAN y Beatriz COCA
}

A new Pre-Pyrenean association of Asplenion petrarchae Br.-Bl. in Meier \& Br.-Bl. 1934 nom. mut.

Palabras clave. Vegetación rupícola, Asplenietea, fitosociología, sintaxonomía, España.

Key words. Rupicolous plant communities, Asplenietea, phytosociology, syntaxonomy, Spain.

Las notables peculiaridades bioclimáticas y biogeográficas de los Prepirineos catalanoaragoneses son bien conocidas desde antiguo (cf. Bolòs, 1957a, 1961). En el caso de las comarcas limítrofes entre Huesca y Lérida, y particularmente en los territorios comprendidos entre las cuencas de los ríos NogueraRibagorzana y Noguera-Pallaresa, al sur de la sierra del Montsec (Lérida), pueden apreciarse cambios significativos en el paisaje en desplazamientos de sólo unos pocos kilómetros.

En estos territorios el bioclima dominante es Mediterráneo pluviestacional oceánicoestépico (Rivas-Martínez \& Loidi, 1999a; Rivas-Martínez et al., 2002) y en él la influencia térmica mediterránea de la Depresión del Ebro penetra hacia el norte por los grandes cauces fluviales y se hace patente casi en los propios contrafuertes pirenaicos, donde las condiciones climáticas son claramente submediterráneas.

Durante la realización de trabajos de cartografía de la vegetación en las áreas prepirenaicas de Huesca y Lérida, tuvimos la oportunidad de inventariar unas formaciones rupícolas dominadas por el endemismo Antirrhinum molle L., que habitan roquedos verticales de naturaleza calcárea y muy térmicos. El estudio de tales inventarios y su comparación con las asociaciones conocidas de la alianza Asplenion petrarchae $\mathrm{Br}$.-B1. in 
Meier \& Br.-Bl. 1934 nom. mut. (Asplenion glandulosi) permiten describir una nueva asociación, hasta el momento endémica de los territorios mencionados.

Las autorías de los táxones mencionados en el texto y tablas corresponden a las que se recogen en Bolòs \& Vigo (1984-2001). Los aspectos bioclimáticos y biogeográficos se ajustan a las indicaciones de Rivas-Martínez \& Loidi (1999a, b) y Rivas-Martínez et al. (2002). La tipología sintaxonómica sigue las propuestas de Rivas-Martínez et al. (2001, 2002), adaptadas al Código Internacional de Nomenclatura Fitosociológica (Weber et al., 2000).

\section{Jasonio saxatilis-Antirrhinetum mollis M.B.} Crespo, Juan \& Coca ass. nov.

(Tabla 1, holotypus inv. 2)

Combinación florística: Asociación caracterizada por la presencia constante y, a menudo, abundante de Antirrhinum molle, Jasonia saxatilis Guss. (三Chiliadenus saxatilis (Lam.) S. Brullo, = J. glutinosa DC.), Galium fruticescens Cav. y Piptatherum coerulescens (Desf.) Beauv., a los que acompañan en mayor o menor grado otros elementos característicos de las unidades superiores -p. ej. Sedum dasyphyllum L. subsp. dasyphyllum, Ficus carica L., Micromeria fruticosa Druce [” Satureja fruticosa (L.) Bég.], Chaenorhinum origanifolium (L.) Fourr. subsp. cadevalli (O. Bolòs \& Vigo) Laínz o Melica minuta L. subsp. minuta-, algunos de los cuales tienen su óptimo en las comarcas del litoral mediterráneo y se encuentran casi finícolas en el área de la asociación. Es destacable asimismo la presencia ocasional de dos endemismos prepirenaicos de distribución semejante a Antirrhinum molle (pero más restringida), los cuales actúan como buenas diferenciales territoriales: Centaurea emigrantis Bubani [ $\equiv$ C. uniflora L. subsp. emigrantis (Bubani) Pau \& Font Quer ex Font Quer], taxon con óptimo en los matorrales seriales de Helianthemo italici-Aphyllanthion monspeliensis Díez Garretas, Fernández-
González \& Asensi 1998, y Linaria bubanii Font Quer [ $\equiv$ L. glauca (L.) Chaz. subsp. bubanii (Font Quer) Valdés], planta a menudo presente también en comunidades de Achnatherion calamagrostis Jenny in Br.-Bl., Roussine \& Nègre 1952 nom. mut.

Ecología y biogeografía: Comunidad casmofítica de escasa cobertura y marcado carácter termófilo, que presenta su óptimo en roquedos verticales o casi verticales, muy soleados y de naturaleza calcárea -básicamente calizas del Cretácico superior y del EocenoSe encuentra principalmente en el piso mesomediterráneo seco, aunque penetra en áreas de tendencia subhúmeda. Hasta ahora se conoce de las comarcas limítrofes entre las provincias de Huesca y Lérida, dentro el sector Somontano Aragonés (subprovincia Bajo Aragonesa, provincia Mediterránea Ibérica Central).

Variabilidad: Se presenta bastante homogénea en todo el territorio estudiado. No obstante, en las zonas más térmicas y secas (invs. 1-4) es donde la asociación se muestra más típica, tomando mayor relevancia táxones xerófilos o termófilos como Rhamnus lycioides L. subsp. lycioides, Lavatera maritima Gouan, Ruta angustifolia Pers. o Ephedrafragilis Desf. Por el contrario, en las áreas más umbrosas y de tendencia subhúmeda (invs. 5-7) desaparecen aquéllas y penetran plantas más exigentes como Buxus sempervirens L., Silene saxifraga L. o Asplenium ruta-muraria $\mathrm{L}$.

\section{Discusión sintaxonómica}

La especie directora de esta nueva asociación, Antirrhinum molle, es un endemismo prepirenaico de amplia valencia ecológica. Se encuentra en distintos ambientes rocosos calcáreos (paredes verticales, roquedos de moderada inclinación e incluso balmas y abrigos extraplomados), habitando indistintamente en áreas expuestas o protegidas, y en territorios climáticamente mediterráneos (pisos mesomediterráneo seco a supramediterráneo sub- 
Tabla 1

Jasonio saxatilis-Antirrhinetum mollis M.B. Crespo, Juan \& Coca ass. nov. (Asplenion petrarchae, Asplenietalia petrarchae, Asplenietea trichomanis)

Orden de inventarios

Inclinación $\left(^{\circ}\right)$

Altitud (m.s.n.m.)

Cobertura (\%)

Área $\left(\mathrm{m}^{2}\right)$

Fecha (mes/año)

\section{Características}

Antirrhinum molle

Jasonia saxatilis

Piptatherum coerulescens

Galium fruticescens

Ficus carica

Sedum dasyphyllum

Lactuca tenerrima

Linaria bubanii (dif.)

Centaurea emigrantis (dif.)

Melica minuta

Micromeria fruticosa

Chaenorhinum cadevalli

Silene saxifraga

Asplenium ruta-muraria

\section{Compañeras}

Polygala rupestris

Fumana ericifolia

Stipa offneri

Sarcocapnos enneaphylla

Thymus vulgaris

Rhamnus lycioides

Lavatera maritima

Ephedra fragilis

Ruta angustifolia

Rosmarinus officinalis

Buxus sempervirens

Helichrysum stoechas

Bupleurum fruticescens

Celtis australis

Juniperus phoenicea

Ononis minutissima

Phagnalon rupestre

$\begin{array}{cccccccc}1 & 2 & 3 & 4 & 5 & 6 & 7 & \text { Indice } \\ 90 \mathrm{~S} & 80 \mathrm{SE} & 90 \mathrm{E} & 90 \mathrm{~S} & 90 \mathrm{~N} & 85 \mathrm{~W} & 80 \mathrm{SW} & \\ 530 & 400 & 360 & 480 & 500 & 500 & 500 & \\ 20 & 15 & 30 & 20 & 20 & 25 & 30 & \\ 5 & 5 & 2 & 4 & 2 & 5 & 4 & \\ \mathrm{X}-02 & \mathrm{X}-02 & \mathrm{X}-02 & \mathrm{X}-02 & \mathrm{X}-02 & \mathrm{X}-02 & \mathrm{X}-02 & \end{array}$

\begin{tabular}{ccccccc|c}
2 & 2 & 3 & 2 & 2 & 2 & 2 & V \\
2 & 1 & 2 & 2 & 1 & 2 & 3 & V \\
+ & + & 1 & 1 & + & + & 2 & V \\
1 & 1 & 1 & + & + & 1 & $\cdot$ & V \\
+ & + & $\cdot$ & + & + & + & + & V \\
$\cdot$ & + & 1 & 1 & $\cdot$ & 2 & 1 & IV \\
+ & + & $\cdot$ & $\cdot$ & $\cdot$ & $\cdot$ & $\cdot$ & II \\
$\cdot$ & $\cdot$ & $\cdot$ & $\cdot$ & $\cdot$ & 1 & $\cdot$ & I \\
$\cdot$ & + & $\cdot$ & $\cdot$ & $\cdot$ & $\cdot$ & $\cdot$ & I \\
$\cdot$ & + & $\cdot$ & $\cdot$ & $\cdot$ & $\cdot$ & $\cdot$ & I \\
$\cdot$ & $\cdot$ & $\cdot$ & + & $\cdot$ & $\cdot$ & $\cdot$ & I \\
$\cdot$ & $\cdot$ & $\cdot$ & $\cdot$ & + & $\cdot$ & $\cdot$ & I \\
$\cdot$ & $\cdot$ & $\cdot$ & $\cdot$ & $\cdot$ & + & $\cdot$ & I \\
$\cdot$ & $\cdot$ & $\cdot$ & $\cdot$ & $\cdot$ & + & $\cdot$ & I \\
& + & & & & & & \\
+ & + & + & + & + & + & + & V \\
+ & $\cdot$ & 1 & + & + & + & + & V \\
+ & + & $\cdot$ & + & $\cdot$ & + & + & IV \\
+ & $\cdot$ & $\cdot$ & + & + & $\cdot$ & + & III \\
$\cdot$ & + & + & $\cdot$ & $\cdot$ & + & + & III \\
+ & + & $\cdot$ & + & $\cdot$ & $\cdot$ & $\cdot$ & III \\
+ & + & $\cdot$ & $\cdot$ & $\cdot$ & $\cdot$ & $\cdot$ & II \\
+ & + & $\cdot$ & $\cdot$ & $\cdot$ & $\cdot$ & $\cdot$ & II \\
$\cdot$ & + & $\cdot$ & + & $\cdot$ & $\cdot$ & $\cdot$ & II \\
$\cdot$ & $\cdot$ & $\cdot$ & + & $\cdot$ & $\cdot$ & + & II \\
$\cdot$ & $\cdot$ & $\cdot$ & $\cdot$ & + & + & + & II \\
$\cdot$ & $\cdot$ & + & $\cdot$ & $\cdot$ & $\cdot$ & $\cdot$ & I \\
$\cdot$ & $\cdot$ & + & $\cdot$ & $\cdot$ & $\cdot$ & $\cdot$ & I \\
$\cdot$ & $\cdot$ & $\cdot$ & + & $\cdot$ & $\cdot$ & $\cdot$ & I \\
$\cdot$ & $\cdot$ & $\cdot$ & + & $\cdot$ & $\cdot$ & $\cdot$ & I \\
$\cdot$ & $\cdot$ & $\cdot$ & + & $\cdot$ & $\cdot$ & + & I \\
$\cdot$ & $\cdot$ & $\cdot$ & $\cdot$ & $\cdot$ & $\cdot$ & & $\cdot$
\end{tabular}

Localidades: 1.- Huesca: Estopiñán, pr. Central Eléctrica de Canelles, CG0150. 2.- Huesca: Castillonroy, hacia la Ermita de Santa Ana, BG9938 (Holotypus ass.). 3.- Huesca: Castillonroy, pr. Central Eléctrica de Santa Ana, BG9939. 4.- Lérida: Santa Linya, Ermita de Sant Miquel, CG1643. 5.- Lérida: Santa Linya, CG 1744. 6.- Lérida: Os de Balaguer, Congost del Río Farfanya, CG1040. 7.- Lérida: Os de Balaguer, Bco. de Alberola, CG0446.

húmedo) o templados (pisos meso-supratemplado subhúmedo). Por esta razón, la JasonioAntirrhinetum muestra ciertas relaciones con la Petrocoptido montsiccianae-Antirrhinetum mollis O. Bolòs 1957 corr. Rivas-Martínez et al. 2002 (alianza Valeriano longifloraePetrocoptidion F. Casas 1972), descrita de territorios colindantes con los aquí estudiados 


\begin{tabular}{lllllcl}
\hline Orden de inventarios & 1 & 2 & 3 & 4 & 5 & 6 \\
$\mathrm{~N}^{\circ}$ inventarios & 7 & 5 & 6 & 7 & 12 & 7 \\
\hline
\end{tabular}

Características de las unidades superiores

Jasonia saxatilis

Piptatherum coerulescens

Ceterach officinarum

Asplenium ruta-muraria

Phagnalon sordidum

Melica minuta

Sedum dasyphyllum

Galium fruticescens

Lactuca tenerrima

Ficus carica

Asplenium petrarchae

Asplenium trichomanes (s.l.)

Mercurialis huetii

Silene saxifraga (transgr.)

Chaenorhinum crassifolium

\begin{tabular}{|c|c|c|c|c|}
\hline I & III & IV & $\mathrm{V}$ & II \\
\hline V & & II & III & \\
\hline I & I & II & r. & V \\
\hline I & II & I & & \\
\hline II & $\cdot$ & I & V & II \\
\hline III & r. & IV & . & V \\
\hline III & . & I & . & . \\
\hline & 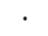 & II & I & . \\
\hline I & $\cdot$ & I & $\cdot$ & I \\
\hline I & $\cdot$ & $\cdot$ &. & IV \\
\hline II & I & . & . & \\
\hline I & & . & . & II \\
\hline$\cdot$ & II & . & 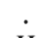 & . \\
\hline . & . & . & II & . \\
\hline
\end{tabular}

Características y diferenciales de asociaciones

Galium lucidum (dif.)

Antirrhinum majus (dif.)

Santolina pecten (dif.)

Centranthus lecoqii (dif.)

Chaenorhinum cadevalli

Campanula affinis (terr.)

Biscutella mediterranea (dif.)

Satureja montana (dif.)

Micromeria fruticosa (terr.)

Ceratonia siliqua (dif.)

Cosentinia vellea (terr.)

Jasione mansanetiana

Satureja innota (dif.)

Antirrhinum litigiosum (dif.)

Cheilanthes acrostica (terr.)

Antirrhinum graniticum (terr.)

Chaenorhinum origanifolium (terr.)

Antirrhinum molle

Centaurea emigrantis (dif.)

Linaria bubanii (dif.)

Procedencia de los inventarios: 1.- Sedo sediformi-Piptatheretum coerulescentis (Bolòs \& Vigo, 1984: 189). 2.- Jasonio glutinosae-Linarietum cadevallii (A. Bolòs \& O. Bolòs, 1950: 61). 3.- Melico minutaeSaturejetum fruticosae (Bolòs \& Vives in O. Bolòs, 1957b: 536). 4.- Phagnalo sordidi-Jasionetum mansanetianae (Roselló, 1994: 389). 5.- Cheilantho acrosticae-Asplenietum petrarchae (Izco, 1970: 94). 6.- Jasonio saxatilis-Antirrhinetum mollis (Tabla 1).

Tabla 2. Sinopsis de las asociaciones iberolevantinas de Asplenion petrarchae.

(Congost de Terradets; cf. Bolòs, 1954); pero, tanto florística como ecológicamente, ambas asociaciones quedan perfectamente independizadas.

Por un lado, la Petrocoptido-
Antirrhinetum se caracteriza por la presencia de los endemismos Petrocoptis montsicciana O. Bolòs \& Rivas Mart. [ $\equiv$ Petrocoptis crassifolia Rouy subsp. montsicciana $(\mathrm{O}$. Bolòs 
\& Rivas Mart.) O. Bolòs \& Vigo] y Brassica repanda (Willd.) DC. subsp. cadevallii (Font Quer) Heyw., a los que acompañan Potentilla caulescens L., Erinus alpinus L., Hieracium amplexicaule L., Ramonda myconii (L.) Rchb., Lonicera pyrenaica L., Thymelaea dioica All., etc. (plantas frecuentes o propias de Saxifragion mediae Br.-Bl. in Meier \& Br.-Bl. 1934 y que están ausentes en la nueva asociación que aquí se propone). Por otro lado, se desarrolla en roquedos extraplomados de cañones y congostos umbrosos, donde las condiciones microclimáticas propician que plantas de pisos altitudinales superiores -como las citadas anteriormente- puedan encontrarse en cotas bajas $(400-500 \mathrm{~m})$, en vecindad con plantas de marcado carácter termófilo que ocupan las solanas, más secas y cálidas (fenómeno que no es raro en áreas limítrofes mediterráneotempladas como la que nos ocupa).

Por su parte, la Jasonio saxatilisAntirrhinetum mollis presenta una composición florística, ecología y ubicación topográfica particulares que permiten reconocerla como un sintaxon independiente en la Asplenion petrarchae, alianza que alcanza su límite de distribución en los territorios prepirenaicos estudiados. En la tabla 2 se muestran comparativamente las relaciones entre las asociaciones iberolevantinas conocidas de dicha alianza, donde puede apreciarse que la caracterización de éstas ha de realizarse en muchas ocasiones atendiendo a sus táxones diferenciales, ya que no disponen de especies características exclusivas.

La presencia ocasional de Chaenorhinum origanifolium subsp. cadevallii (= Linaria origanifolia subsp. flexuosa) y Micromeria fruticosa ( $\equiv$ Satureja fruticosa) en nuestros inventarios podría llevar a relacionarla respectivamente con las asociaciones JasonioLinarietum cadevalli (de óptimo vallesanoempordanés litoral) y Melico-Saturejetum fruticosae (de amplia distribución vallesanoempordanesa y valenciano-tarraconense). Sin embargo, debe tenerse en cuenta que ambos táxones muestran una distribución amplia en el noreste y este peninsulares, por lo que su mera presencia no debería utilizarse con carácter diagnóstico (nótese que Chaenorhinum origanifolium subsp. cadevalli está presente también en la tabla original de la MelicoSaturejetum). Por el contrario, algunos otros táxones sí ofrecen información territorial al respecto; es el caso de Campanula affinis Schultes o Biscutella mediterranea Jord. [ $[\equiv B$. laevigata L. subp. mediterranea (Jord.) Braun. Blanq. et al.] en la Jasonio-Linarietum, o Cosentinia vellea (Aiton) Tod. y Ceratonia siliqua L. en la Melico-Saturejetum.

Las relaciones con otras asociaciones de Asplenion petrarchae son anecdóticas y la presencia de algunos endemismos locales que actúan como características o diferenciales (p. ej. Santolina chamaecyparissus L. subsp. pecten Rouy o Antirrhinum majus L. en la Sedo-Piptatheretum, de distribución vallesanoempordanesa septentrional; Jasione mansanetiana Roselló \& Peris, Antirrhinum litigiosum Pau o Satureja innota (Pau) Pau ex Zapater en la Phagnalo-Jasionetum, valenciano-tarraconense; y Antirrhinum graniticum Roth. subsp. graniticum o Chaenorhinum origanifolium subsp. origanifolium en la Cheilantho-Asplenietum, manchego-sagrense) permiten una fácil discriminación. Como se ha dicho, la constancia de Antirrhinum molle junto con la presencia de Centaurea emigrantis y Linaria badalii garantizan la independencia de la nueva asociación.

\section{Esquema sintaxonómico de las comunidades iberolevantinas de Asplenion petrarchae}

ASPLENIETEA TRICHOMANIS (Br.-Bl. in Meier \& Br.-B1. 1934) Oberdorfer 1977

+ Asplenictalia petrarchae Br.-Bl. in Meier \& Br.B1. 1934, nom. mut.

* Asplenion petrarchae Br.-Bl. in Meier \& Br.-Bl. 1934, nom. mut.

1. Sedo-Piptatheretum coerulescentis O. Bolòs \& 
Vigo 1984 [Asociación vallesano-empordanesa septentrional, con óptimo en roquedos verticales soleados de áreas litorales mesomediterráneas].

2. Jasonio glutinosae-Linarietum cadevallii A. \& O. Bolòs 1950 corr. O. Bolòs 1967 [Asociación vallesano-empordanesa meridional y con irradiaciones bajo-aragonesas, con óptimo en roquedos verticales sombreados de áreas litorales mesomediterráneas].

3. Melico minutae-Saturejetum fruticosae $\mathrm{O}$. Bolòs \& Vives in O. Bolòs 1957 [Asociación vallesano-empordanesa meridional y valenciano-tarraconense, con óptimo en roquedos soleados y a menudo con poca inclinación, de áreas litorales termo-mesomediterráneas].

4. Phagnalo sordidi-Jasionetum mansanetianae Roselló 1994 [Asociación valencianotarraconense central, con óptimo en roquedos verticales sombreados, de áreas litorales mesomediterráneas].

5. Cheilantho acrosticae-Asplenietum petrarchae Izco 1970 corr. Rivas-Martínez 1983 [Asociación manchego-sagrense, con óptimo en roquedos verticales soleados, de áreas mesomediterráneas].

6. Jasonio saxatilis-Antirrhinetum mollis M.B. Crespo, Juan \& Coca ass. nov. [Asociación somontano-aragonesa, con óptimo en roquedos verticales soleados, de áreas prepirenaicas mesomediterráneas].

\section{BIBLIOGRAFÍA}

BOLÒS, A. DE \& O. DE BOLÒS - 1950- Vegetación de las comarcas Barcelonesas. Instituto Español de Estudios Mediterráneos. Barcelona.

BOLÒS, O. DE -1954- De Vegetatione notulae I. Collect. Bot. (Barcelona) 4(2): 253-286.

BOLÒS, O. DE -1957a- Les zones de vegetació de Catalunya. Societat catalana de Geografia. Institut d'Estudis Catalans. Barcelona.

BOLÒS, O. DE -1957b- De vegetatione valentina I. Collect. Bot. (Barcelona) 5(2): 527-599.

BOLÒS, O. DE -1961- La transición desde la Depresión del Ebro y los Pirineos en el aspecto geobotánico. Anales Inst. Bot. Cavanilles 18: 199-254.

BOLÒS, O. DE - 1967- Comunidades vegetales de las comarcas próximas al litoral situadas entre los ríos Lobregat y Segura. Mem. Real Acad. Ci. Barcelona 38(1): 3-281.

BOLÒS, O. DE \& J. VIGO - 1984- Flora vascular i vegetació de les Illes Medes. Arxius Secc. Ci. Inst. Estud. Catalans 73: 131-208.

BOLÒS, O. DE \& J. VIGO - 1984/2001- Flora dels Països Catalans. 4 vols. Barcelona.

IZCO, J. - 1970- Elementos y comunidades térmicomediterráneos en la planicie Carpetana. Anales Inst. Bot. Cavanilles 26: 89-101.

RIVAS-MARTÍNEZ, S., F. FERNÁNDEZ GONZÁLEZ, J. LOIDI, M. LOUSÃ \& A. PENAS -2001- Syntaxonomical checklist of vascular plant communities of Spain and Portugal to association level. Itinera Geobot. 14: 1-341.

RIVAS-MARTÍNEZ, S., T. E. DÍAZ, F. FERNÁNDEZGONZÁLEZ, J. IZCO, J. LOIDI, M. LOUS Ã \& A. PENAS -2002-Vascular plant communities of Spain and Portugal. Addenda to the Syntaxonomical Checklist of 2001. Itinera Geobot. 15(1-2): 5-922.

RIVAS MARTÍNEZ, S. \& J. LOIDI -1999aBioclimatology of the Iberian Peninsula. In: RIVAS-MARTÍNEZ, S. et al. (eds.), Iter ibericum A.D. MIM (Excursus geobotanicus per Hispaniam et Lusitaniam, ante XLII Symposium Societatis Internationalis Scientiae Vegetationis Bilbao mense Iulio celebrandum dicti Anni). Itinera Geobot. 13: 41-47.

RIVAS MARTÍNEZ, S. \& J. LOIDI -1999bBiogeography of the Iberian Peninsula. In: RIVAS-MARTÍNEZ, S. et al. (eds.), Iter ibericum A.D. MIM (Excursus geobotanicus per Hispaniam et Lusitaniam, ante XLII Symposium Societatis Internationalis Scientiae Vegetationis Bilbao mense Iulio celebrandum dicti Anni). Itinera Geobot. 13: 49-67.

ROSELLÓ, R. -1994- Catálogo florístico y vegetación de la comarca natural del Alto Mijares. Excma. Diputación de Castellón.

WEBER, H. E., MORA VEC, J. \& THÉURILLAT, J. P. -2000- International Code of Phytosociological Nomenclature. $3^{\text {rd }}$ edition. J. Veg. Sci. 11: 739768.

Aceptado para su publicación en enero de 2003

Dirección de los autores. Centro Iberoamericano de Biodiversidad (CIBIO). Universidad de Alicante. Apdo.99.E-03080 Alicante.E-mail: crespo@ua.es 